\title{
Terahertz four-wave mixing spectroscopy for study of ultrafast dynamics in a semiconductor optical amplifier
}

\author{
Jianhui Zhou, Namkyoo Park, Jay W. Dawson, and Kerry J. Vahala \\ Department of Applied Physics, Mail Stop 128-95, California Institute of Technology, Pasadena, \\ California 91125 \\ Michael A. Newkirk and Barry I. Miller \\ AT\& T Bell Laboratories, Holmdel, New Jersey 07733
}

(Received 2 April 1993; accepted for publication 18 June 1993)

\begin{abstract}
Ultrafast dynamics in a $1.5-\mu \mathrm{m}$ tensile-strained quantum-well optical amplifier has been studied by highly nondegenerate four-wave mixing at detuning frequencies up to $1.7 \mathrm{THz}$. Frequency response data indicate the presence of two ultrafast physical processes with characteristic relaxation lifetimes of $650 \mathrm{fs}$ and $<100 \mathrm{fs}$. The longer time constant is believed to be associated with the dynamic carrier heating effect. This is in agreement with previous time-domain pump-probe measurements using ultrashort optical pulses.
\end{abstract}

Understanding the ultrafast intraband dynamics of semiconductor active layers is of considerable importance since, in addition to purely fundamental considerations, the physical processes involved influence the modulation response and spectral properties of semiconductor lasers and can induce crosstalk between multiplexed signals in semiconductor optical amplifiers.

Until recently, time-domain pump-probe measurement using ultrashort optical pulses, ${ }^{1-5}$ pioneered by Ippen and co-workers, ${ }^{1-3}$ has been the only direct technique for study of ultrafast intraband dynamics. These measurements have revealed characteristic time constants of 650 and $200 \mathrm{fs}$ in $1.5 \mu \mathrm{m}$ InGaAsP bulk optical amplifiers ${ }^{1}$ and 700 and 250 fs in $1.5 \mu \mathrm{m}$ multiple quantum-well optical amplifiers. ${ }^{2}$ The longer time constants were attributed to a heated carrier distribution cooling back to an equilibrium temperature and is referred to as dynamic carrier heating. The shorter time constants were recently found to be caused by a combination of effects, including delay in the carrier heating, and spectral hole burning. ${ }^{3}$ In addition, measurements on multiple quantum-well optical amplifiers by Weiss et al. ${ }^{4}$ have resolved time constants of $2-7 \mathrm{ps}$, which were attributed to carrier diffusion in the barrier layer and identified as the carrier capture time.

Highly nondegenerate four-wave mixing due to ultrafast intraband dynamics in semiconductor gain media was first proposed by Agrawal. ${ }^{6}$ Recently, highly nondegenerate four-wave mixing measurements in semiconductor traveling-wave optical amplifiers (TWAs) have been used as a frequency-domain technique for studies of ultrafast dynamics in semiconductors. To date, however, all the measurements have been limited to a detuning range of approximately $500 \mathrm{GHz} .{ }^{7-9}$ Here, we report measurements of four-wave mixing in a tensile-strained multiple quantum-well TWA at detuning frequencies up to 1.7 $\mathrm{THz}$, which corresponds to an equivalent temporal resolution of $94 \mathrm{fs}(\tau=1 / 2 \pi f)$. To our knowledge, this represents the first report of terahertz four-wave mixing (TFWM) spectroscopy in semiconductor gain media.

TFWM is a small-signal technique since semiconductor dynamics is probed using optical powers typically in the range of several hundred microwatts. This is in contrast to time-domain pump-probe measurements, where short pulse peak power is usually in the range of several hundred milliwatts or higher. ${ }^{4,5}$ The information revealed in TFWM measurements is therefore readily applicable to analysis of modulation and spectral properties of semiconductor lasers, where small-signal data are often needed. Among other advantages of TFWM are the simplicity of the experimental setup and better accuracy in measurement of lifetime data since short lifetimes are mapped to a wide frequency span. In addition to providing a frequencydomain tool for investigation of intraband dynamics, TFWM measurements provide signal spectra that give amplifier crosstalk strength for widely separated optical channels as well as efficiency for possible use of TWAs as parametric channel converters.

The TWA used in this study was a tensile-strained InGaAs/InGaAsP multiple quantum-well TWA operating at $1.5 \mu \mathrm{m}$. Details on the structure appear in Refs. 10 and 11. The specific device was operated at a $100 \mathrm{~mA}$ bias current where the TM mode small-signal gain was measured to be $20 \mathrm{~dB}$ for the wavelengths investigated, and the gain ripple less than $0.1 \mathrm{~dB}$. The pump, probe, and local oscillators used in the measurements are three singlefrequency, tunable, Er-doped fiber ring lasers. ${ }^{12}$ These lasers, having output powers of about $1 \mathrm{~mW}$, linewidths less than $4 \mathrm{kHz},{ }^{13}$ and low intensity noise levels, ${ }^{14}$ provide ideal sources for the TFWM experiment.

As shown in Fig. 1, the optical outputs from the pump and probe lasers, having optical frequencies $f_{p}$ and $f_{q}$, were combined using a $3 \mathrm{~dB}$ fiber coupler, and then collimatcd and coupled into the TWA using microscope objectives. The optical power coupled into the TWA was about $250 \mu \mathrm{W}$. Two fiber polarization controllers were employed to match the polarization state of input pump and probe to the TWA TM mode. Two optical isolators providing $60 \mathrm{~dB}$ isolation were placed before and after the TWA to eliminate feedback caused by the facets of the input and output fibers, and to also serve as polarization selectors.

During the measurements, the pump laser $\left(f_{p}\right)$ was fixed at $1534 \mathrm{~nm}$, while the probe $\left(f_{q}\right)$ was tuned to vary 


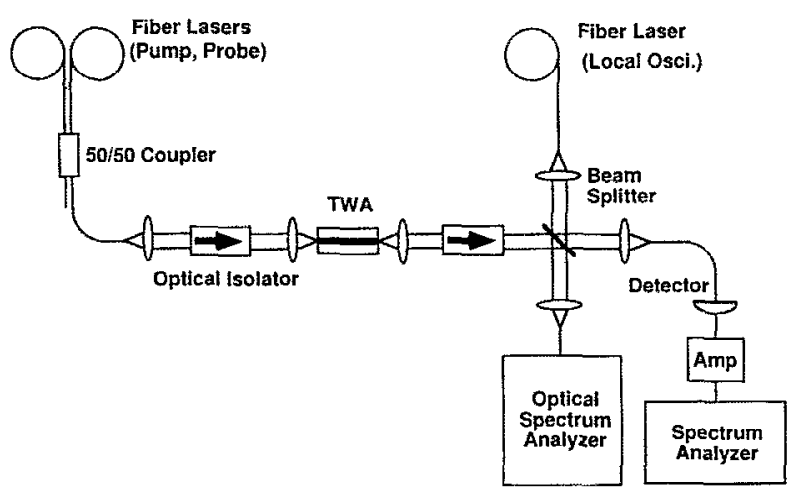

FIG. 1. Experimental setup for the TFWM measurement

the detuning frequency $f_{p}-f_{q}$. The four-wave mixing signal at $2 f_{p}-f_{q}$ was heterodyne detected by mixing the signal with the fiber-laser local oscillator and tuning the local oscillator near the signal frequency. The mixing frequency was maintained at $4.00 \mathrm{GHz}$ throughout the measurements to eliminate the frequency dependence of detection electronics. The detected photocurrent was amplified by a microwave amplifier with $40 \mathrm{~dB}$ gain and then measured using a spectrum analyzer. The detuning frequencies were determined using an optical spectrum analyzer. The optical powers of pump, probe, and local oscillator were also measured, so that the measured four-wave mixing signal could be normalized.

The four-wave mixing signal was measured for detuning frequencies from -1.7 to $1.5 \mathrm{THz}$, limited only by the tunability of the fiber lasers used in the experiment. The signal level at the maximum detuning frequency was still about $20 \mathrm{~dB}$ above the noise floor, owing to the narrow linewidth and low intensity noise of the fiber lasers. The normalized signal power as a function of detuning frequency is illustrated in Figs. 2 and 3.

In prior work at lower frequencies, we have been able to model these effects using uncoupled rate equations characterized by a lifetime and a complex coupling coefficient. ${ }^{8,9}$ To study the new ultrahigh-frequency data we

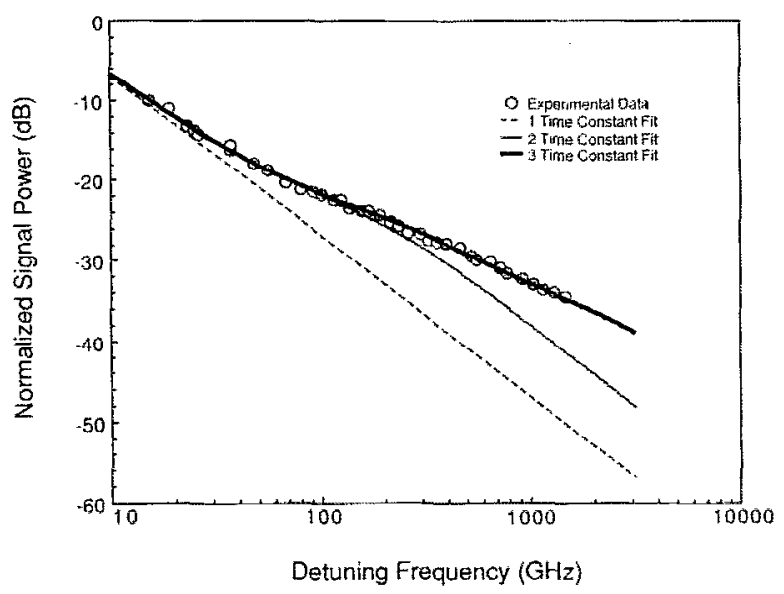

FIG. 2. Normalized four-wave mixing signal power vs positive detuning frequency showing theoretical fits.

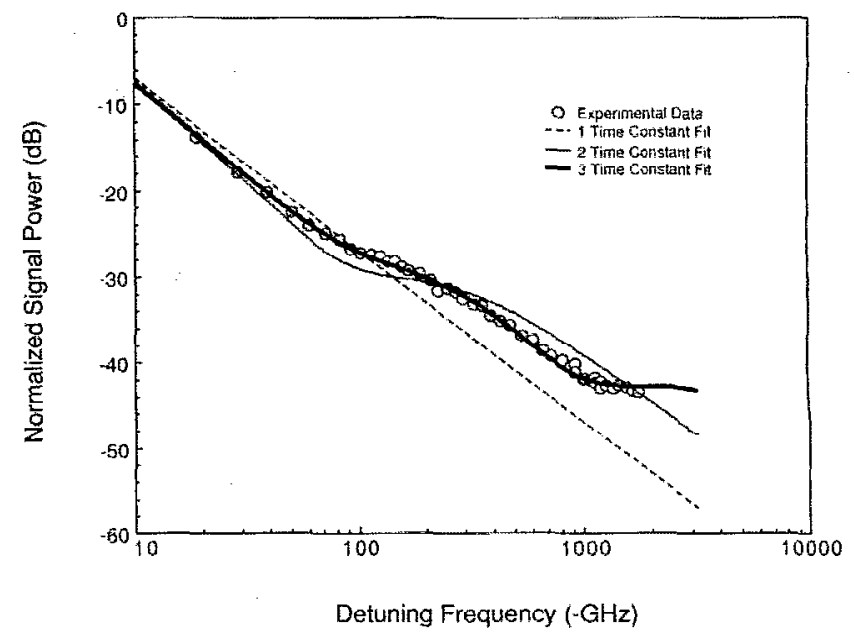

FIG. 3. Normalized four-wave mixing signal power vs negative detuning frequency showing theoretical fits.

have extended the simplified formula to include the possibility of several physical processes,

$$
E_{\mathrm{FWM}}=E_{p}^{2} E_{q}^{*} \sum_{j} \frac{c_{j}}{1-i 2 \pi f \tau_{j}},
$$

where $E_{\mathrm{FWM}}, E_{p}$, and $E_{q}$ are amplitudes of the signal, pump, and probe fields, $f$ is the detuning frequency, and $\tau_{j}$ and $c_{j}$ are the lifetimes and the complex coupling coefficients of the various relaxation processes. Physically, the real and imaginary parts of the coupling coefficient represent the strengths of the dynamic gain and index gratings created by the specific relaxation process. Therefore, the coupling coefficients can serve, in some cases, as a criterion for identifying the responsible physical mechanisms.

The dashed line in Figs. 2 and 3 represents the response accounting for carrier density modulation (interband recombination) only. The deviation of the measured signal data from this one time constant fit indicates the presence of the ultrafast relaxation mechanisms. In addition, the asymmetric nature of the signal spectrum is believed to be caused by phase interferences which occur between various mechanisms present. Since the positive detuning data show constructive interferences, we estimated a second time constant (i.e., in addition to the interband recombination time constant) using the positive signal spectrum. However, a two time constant response is still insufficient to provide a good fit for the whole signal spectrum as shown in the figures. This indicates the existence of an even faster process. A third term having a lifetime of $<100 \mathrm{fs}$ was required to obtain a good fit.

Using the three-term formula, excellent fits were obtained for both positive and negative detuning data with the same parameter set (see Figs. 2 and 3:-3 time constant fit). The fitting parameters are as follows: $c_{1}$ $=5.65 e^{-i 1.30}, c_{2}=0.0642 e^{i 1.30}, c_{3}=0.0113 e^{i 1.53}, \tau_{1}=200 \mathrm{ps}$, $\tau_{2}=650 \mathrm{fs}$, and $\tau_{3}=50 \mathrm{fs}$. Here we have used a value of 3.6 for the linewidth enhancement factor. It is important to note that the fits are quite sensitive to the longer intraband relaxation time constant $650 \mathrm{fs}$, but are relatively insensi- 
tive to the shorter time constant, because the equivalent temporal resolution of this measurement system is still limited.

As pointed out earlier in this letter, the real and imaginary parts of the coupling coefficient represent the strengths of the dynamic gain and index gratings created by the specific relaxation process. The complex coupling coefficient $c_{2}$ gives a value of -3.6 for the ratio of real and imaginary parts of the refractive index change induced by the mechanism having a lifetime of $650 \mathrm{fs}$. This value is qualitatively consistent with dynamic carrier heating (the value would be nearly zero for the case of spectral hole burning). The time constant of $650 \mathrm{fs}$, itself, is in good agreement with the results of previous measurements of dynamic carrier heating in both time and frequency domains. ${ }^{1-5,7}$

Since the equivalent temporal resolution of this study is limited to about $100 \mathrm{fs}$, there is some uncertainty in determining the shorter time constant, and consequently, there exists uncertainty in the corresponding coupling coefficient. The available data are therefore insufficient to make accurate statements concerning the physical mechanisms associated with the shorter lifetime. However, it may be related to other proposed mechanisms, such as initial carrier equilibration dynamics and spectral hole burning.

In conclusion, we have presented the first TFWM measurement on a semiconductor optical amplifier. The fourwave mixing signal was measured as a function of detuning frequency from -1.7 to $1.5 \mathrm{THz}$, which provides a $94 \mathrm{fs}$ equivalent temporal resolution. The time constant 650 is determined in the measurement is in good agreement with the results of previous time-domain measurements using ultrashort optical pulses. The experimental data also indicate the presence of an ultrafast process with a lifetime $<100 \mathrm{fs}$. The maximum detuning frequency in this measurement was limited only by the fiber laser tuning range.
With improvements to the fiber lasers, the detuning frequency could be further extended to provide even better temporal resolutions. Finally, with more measurements and better modeling, TFWM will become a very important technique for frequency-domain investigation of ultrafast dynamics in semiconductor laser devices.

This work was supported in part by the Office of Naval Research under Contract No. No0014-91-1524, and by Northrop Corporation. The authors would also like to thank Dr. Doug Hall of Corning Inc. for critical assistance in the construction of the Er-doped fiber amplifiers used in this experiment.

${ }^{1}$ K. L. Hall, J. Mark, E. P. Ippen, and G. Eisenstein, Appl. Phys. Lett. 56, 1740 (1990).

${ }^{2}$ K. L. Hall, Y. Lai, E. P. Ippen, G. Eisenstein, and U. Koren, Appl, Phys, Lett. 57, 2888 (1990).

${ }^{3}$ K. L. Hall, G. Lenz, E. P. Ippen, U. Koren, and G. Raybon, Appl. Phys. Lett, 61, 2512 (1992).

${ }^{4}$ S. Weiss, J. M. Wiesenfeld, D. S. Chemla, G. Raybon, G. Sucha, M. Wegener, G. Eisenstein, C. A. Burrus, A. G. Dentai, U. Koren, B. I. Miller, H. Temkin, R. A. Logan, and T. Tanbun-Ek, Appl. Phys. Lett. 60, 9 (1992).

${ }^{5}$ J. Mark and J. Mörk, Appl. Phys. Lett. 61, 2281 (1992).

${ }^{6}$ G. P. Agrawal, J. Opt. Soc. Am. B 5, 147 (1988).

${ }^{7}$ L. F. Tiemeijer, Appl. Phys. Lett. 59, 499 (1991).

${ }^{8}$ K. Kikuchi, M. Kakui, C. E. Zah, and T. P. Lee, IEEE J. Quantum Electron. QE-28, 151 (1992)

${ }^{9}$ J. Zhou, N. K. Park, J. Dawson, K. J. Vahala, M. A. Newkirk, U. Koren, and B. I. Miller, Appl. Phys. Lett. 62, 230 I (1993).

${ }^{10}$ M. A. Newkirk, U. Koren, B. I. Miller, M. D. Chien, M. G. Young, T. L. Koch, G. Raybon, C. A. Burrus, B. Tell, and K. F. Brown-Goebeler, IEEE Photon. Technol. Lett. 4, 1258 (1992).

"B. I. Miller, U. Koren, M. A. Newkirk, M. G. Young, R. M. Jopson, R. M. Derosier, and M. D. Chien, IEEE Photon. Technol. Lett. 5, 520 (1993).

${ }^{12}$ N. K. Park, J. W. Dawson, K. J. Vahala, and C. Miller, Appl. Phys. Lett. 59, 2369 (1991).

${ }^{13}$ N. K. Park, J. W. Dawson, and K. J. Vahala, Opt. Lett. 178, 1274 (1992).

${ }^{14}$ S. Sanders, N. K. Park, J. W. Dawson, and K. J. Vahala, Appl. Phys, Lett. 60, 2583 (1992). 